\title{
Multiplex Real-Time PCR Assays for the Identification of the Potato Cyst and Tobacco Cyst Nematodes
}

\author{
Mark K. Nakhla, Kristina J. Owens, Wenbin Li, and Gang Wei, National Plant Germplasm and Biotechnology \\ Laboratory, United States Department of Agriculture (USDA) Animal and Plant Health Inspection Service (APHIS) \\ PPQ-CPHST; Andrea M. Skantar, Nematology Laboratory, USDA Agricultural Research Service; and Laurene \\ Levy, National Plant Germplasm and Biotechnology Laboratory, USDA-APHIS-PPQ-CPHST, Beltsville, MD 20705
}

\begin{abstract}
Nakhla, M. K., Owens, K. J., Li, W., Wei, G., Skantar, A. M., and Levy, L. 2010. Multiplex realtime PCR assays for the identification of the potato cyst and tobacco cyst nematodes. Plant Dis. 94:959-965.

TaqMan primer-probe sets were developed for the detection and identification of potato cyst nematodes (PCNs) Globodera pallida and G. rostochiensis using two-tube, multiplex real-time polymerase chain reaction (PCR). One tube contained a primer-probe set specific for G. pallida (pale potato cyst nematode) multiplexed with another primer-probe set specific for G. rostochiensis (golden potato cyst nematode). A second tube consisted of the G. pallida-specific primer-probe set multiplexed with a primer-probe set specific for G. tabacum (the morphologically similar tobacco cyst nematode). This internal transcribed spacer rDNA-based system was specific for the Globodera spp. of interest and successfully identified several populations of PCN. This rapid, sensitive, and specific quantitative PCR assay presents a useful tool for PCN regulatory response and management programs.
\end{abstract}

The pale potato cyst nematode Globodera pallida (Stone, 1973) Behrens, 1975 and the golden potato cyst nematode $G$. rostochiensis (Wollenweber, 1923) Behrens, 1975 are regulated pathogens of potato (Solanum tuberosum) in the United States. Previously, the distribution of $G$. pallida in North America was limited to Newfoundland, Canada but, in 2006, G. pallida was discovered in northern Bingham County, ID (7). The presence of this nematode represents a significant threat to the U.S. potato industry; therefore, accurate identification of Globodera spp. found in North America is essential for rational regulatory decisions to eradicate and prevent the spread of potato cyst nematodes (PCNs).

$G$. pallida and $G$. rostochiensis are pathogens of potato ( $S$. tuberosum) and other closely related species in the family Solanaceae. The tobacco cyst nematode, G. tabacum (Lownsbery and Lownsbery,

Corresponding author: M. K. Nakhla E-mail: Mark.Nakhla@aphis.usda.gov

Mention of trade names or commercial products in this publication is solely for the purpose of providing specific information and does not imply recommendation or endorsement by the United States Department of Agriculture.

Accepted for publication 7 April 2010.

\section{doi:10.1094/PDIS-94-8-0959}

This article is in the public domain and not copyrightable. It may be freely reprinted with customary crediting of the source. The American Phytopathological Society, 2010.
1954) Behrens, 1975 is a third species of Globodera that can parasitize members of the Solanaceae family. Although it is not a known pathogen of potato, the G. tabacum complex contains three subspecies that, in nature, parasitize solanaceous weeds $(G$. tabacum virginiae) and both tobacco and tomato ( $G$. tabacum tabacum and G. tabacum solanacearum) (2,25). Within North America, G. tabacum is found in the states of Virginia, Connecticut, Massachusetts, and North Carolina, as well as in Mexico and Quebec, Canada $(4,13,14,25)$. The morphological similarity between $G$. tabacum and G. pallida can make identification based on these features alone very difficult $(2,6,15-17)$. This complication led to the development of species-specific molecular assays to separate G. pallida, $G$. rostochiensis, and G. tabacum (23).

In order to determine the extent of $G$. pallida infestation in the United States, federal, state, and local authorities have initiated voluntary potato field surveys and monitoring programs in Idaho and other potato-producing states. Processing of soil samples to extract the nematodes and preliminary morphological characterization of suspect PCN samples is typically performed by state or regional facilities. Isolated nematode cysts suspected of being PCN are typically sent to United States Department of Agriculture (USDA) confirmation laboratories at Beltsville, MD for final identification based on both morphometric characterization and established molecular assays, including conventional polymerase chain reaction (PCR) and restriction fragment length polymorphism analysis $(18,23)$. These tools are capable of identifying PCNs (G. pallida and G. rostochiensis) and distinguishing PCNs from the tobacco cyst nematode G. tabacum. One disadvantage of the current identification scheme is that the entire process is rather labor intensive and takes a minimum of 2 days to complete. Thus, the rationale for this study was to develop a fast and reliable confirmation method, particularly for use by diagnostic laboratories that need to provide results as quickly as possible.

Real-time, quantitative PCR (qPCR) has gained acceptance for pathogen identification due to its improved speed, sensitivity, reproducibility, robustness and the reduced risk of carryover contamination compared with conventional PCR (11). Several qPCR assays have been developed for simultaneous detection of $G$. pallida and the sugarbeet cyst nematode Heterodera schachtii (12) as well as for identification of $G$. pallida and $G$. rostochiensis $(1,3,22)$. These assays are rapid and specific but do not detect G. tabacum. In this study, we present, for the first time, the development of multiplex TaqMan qPCR for the simultaneous detection and identification of the two known PCN species and the morphologically similar tobacco cyst nematode. Because our molecular diagnostic laboratory receives nematode specimens already isolated from soil or plant material, we developed and optimized our molecular diagnostic tool for use on these isolated nematode specimens.

\section{MATERIALS AND METHODS}

Nematode materials. Three established Globodera spp. populations were used for the development of the multiplex TaqMan qPCR. G. pallida cysts that had been previously collected from infested fields in Idaho were kindly provided by Eoin Davis (USDA Animal and Plant Health Inspection Service PPQ, Idaho Falls, ID). G. rostochiensis cysts were kindly provided by Dr. Xiaohong Wang (USDA Agricultural Research Service, Ithaca, NY) and $G$. tabacum solanacearum cysts were kindly provided by Dr. Jon Eisenback (Virginia Tech University, Blacksburg, VA). Specimens from other populations of plant parasitic nematodes of Globodera spp., Heterodera spp., Meloidogyne spp., Nacobbus aberrans, and Pratylenchus penetrans 
were used for validation of the qPCR (Table 1).

DNA extraction. Two alternative methods were used for nematode DNA extraction. For the "single-worm smash" extraction method, each juvenile was visualized with a Nikon SMZ645 dissecting microscope (Nikon, Melville, NY) and mechanically disrupted with an eye-knife in $20 \mu \mathrm{l}$ of nematode extraction (NE) buffer (10 $\mathrm{mM}$ Tris [pH 8.2], $2.5 \mathrm{mM} \mathrm{MgCl} 2,50 \mathrm{mM}$ $\mathrm{KCl}, 0.45 \%$ Tween 20 , proteinase $\mathrm{K}$ at 60 $\mu \mathrm{g} / \mathrm{ml}$, and $0.0005 \%$ gelatin) (26). To reduce the potential of cross contamination during PCR analysis, this step was performed in a separate location from the molecular diagnostic laboratory. Alternatively, the "rapid nematode-DNA" extraction method was developed for rapid processing of samples obtained for PCN detection, as part of the federal and state surveys. Individual cysts or juveniles in 50 $\mu l$ of double-distilled water were loaded into 2.0-ml screwcap microtubes (Sarstedt, Inc., Newton, NC). This is normally the way samples (from federal and state surveys) are received after morphological identification. Equal volumes of $2 \times \mathrm{NE}$ buffer were added to the samples, then stored at $-80^{\circ} \mathrm{C}$ for at least $30 \mathrm{~min}$. Frozen nematodes were thawed and ground thoroughly using the Mini-Beadbeater-1 (BioSpec Products, Inc., Bartlesville, OK) with $5 \mathrm{~mm}$ of glass beads (approximately $30 \mathrm{~s}$ ); then, $2.4 \mu \mathrm{l}$ of proteinase $\mathrm{K}$ was added to each tube. Tubes were incubated for proteinase $\mathrm{K}$ treatment at $60^{\circ} \mathrm{C}$ for $60 \mathrm{~min}$, followed by deactivation of the enzyme by incubation at $95^{\circ} \mathrm{C}$ for $15 \mathrm{~min}$. The nematode extracts were used directly in sequence-specific multiplex qPCR and conventional PCR. DNA extractions from single, 10, 50, or 100 juveniles (or eggs from a cyst) or 1 cyst of each of three Globodera spp. (G. pallida, G. rostochiensis, and $G$. tabacum) in a minimum of three replicates were used for validation of the assay. Nematode DNA extracts $(2 \mu \mathrm{l})$ prepared by either extraction method were used for each qPCR, and $5 \mu$ of the extract was used for each conventional PCR.

Primer and probe design. Globodera spp. rDNA sequences (including multiple sequences for individual species) were downloaded from GenBank (http://www. ncbi.nlm.nih.gov). The sequences (Table 2) were then aligned using DNAMAN Sequence Analysis Software (version 6.0.3 95; Lynnon Corporation, VauderuilDorion, Quebec, Canada). Internal transcribed spacer (ITS)1 region sequences that are conserved within each species were identified visually and used to design three new species-specific primer sets and two new TaqMan probes (Table 3). We developed a set of primers (PITSpf and PITS4) and a TaqMan probe (GFAMp) for the specific detection of G. pallida, and

Table 1. Average cross-threshold $(\mathrm{Ct})$ values for single juveniles extracted using the single-worm smash method

\begin{tabular}{|c|c|c|c|c|c|}
\hline \multirow[b]{3}{*}{ Species } & \multirow[b]{3}{*}{ Origin } & \multicolumn{4}{|c|}{ Ct value } \\
\hline & & \multicolumn{2}{|c|}{ Tube A } & \multicolumn{2}{|c|}{ Tube B } \\
\hline & & FAM & TET & FAM & TET \\
\hline \multirow{7}{*}{ Globodera pallida } & Idaho, United States & 30.01 & 0 & 29.73 & 0 \\
\hline & Switzerland & 27.49 & 0 & 27.80 & 0 \\
\hline & Peru ${ }^{a}$ & 26.84 & 0 & 27.09 & 0 \\
\hline & Peru $^{b}$ & 27.16 & 0 & 27.14 & 0 \\
\hline & England, United Kingdom & 26.21 & 0 & 26.62 & 0 \\
\hline & Netherlands & 26.67 & 0 & 27.08 & 0 \\
\hline & Chile & 28.73 & 0 & 28.87 & 0 \\
\hline \multirow[t]{4}{*}{ G. rostochiensis } & New York, United States & 0 & 30.33 & 0 & \\
\hline & British Columbia, Canada & 0 & 31.74 & 0 & 0 \\
\hline & British Columbia, Canada & 0 & 33.03 & 0 & 0 \\
\hline & York, United Kingdom & 0 & 32.89 & 0 & 0 \\
\hline \multirow[t]{3}{*}{ G. tabacum solanacearum } & Dinwiddie Co., Virginia, & & & & \\
\hline & United States & 0 & 0 & 0 & 28.75 \\
\hline & Richmond, Virginia, United States & 0 & 0 & 0 & 27.17 \\
\hline \multirow[t]{5}{*}{ G. tabacum tabacum } & Connecticut, United States & 0 & 0 & 0 & 27.47 \\
\hline & Virginia, United States & 0 & 0 & 0 & 29.16 \\
\hline & Warner Co., Virginia, & & & & \\
\hline & United States & 0 & 0 & 0 & 29.12 \\
\hline & Virginia, United States & 0 & 0 & 0 & 28.04 \\
\hline Globodera sp. & Oregon, United States & 0 & 0 & 0 & 0 \\
\hline Heterodera avenae & Idaho, United States & 0 & 0 & 0 & 0 \\
\hline H. glycines & Maryland, United States & 0 & 0 & 0 & 0 \\
\hline H. lespedezae & Egypt & 0 & 0 & 0 & 0 \\
\hline M. chitwoodi & Oregon, United States & 0 & 0 & 0 & 0 \\
\hline M. hapla & Hawaii, United States & 0 & 0 & 0 & 0 \\
\hline M. naasi & Oregon, United States & 0 & 0 & 0 & 0 \\
\hline M. arenaria & Virginia, United States & 0 & 0 & 0 & 0 \\
\hline Nacobbus aberrans & Peru & 0 & 0 & 0 & 0 \\
\hline Pratylenchus penetrans & Wisconsin, United States & 0 & 0 & 0 & 0 \\
\hline
\end{tabular}

also developed another set of primers (PGrtf and Prostor) and a TaqMan probe (GTETp) for the specific detection of $G$. rostochiensis. For the specific detection of G. tabacum, we again utilized the GTETp TaqMan probe and developed a third primer set (PGrtf and PITSt3mr). Primers and TaqMan probes were analyzed using OligoAnalyzer (version 3.1; http://www. idtdna.com/analyzer/Applications/OligoAn alyzer/) prior to synthesis by Integrated DNA Technologies, Inc. (Coralville, IA).

Real-time PCR protocol. TaqMan qPCR assays were performed in a SmartCycler II (Cepheid, Sunnyvale, CA) in a $25-\mu$ reaction volume. We tested each sample in two tubes (tube A and tube B). Tube A (Fig. 1) contained the G. pallidaspecific set (primer PITSpf, primer PITSp4, and the FAM-labeled TaqMan probe GFAMp) multiplexed with the $G$. rostochiensis-specific set (primer PGrtf, primer Prostor, and the TET-labeled TaqMan probe GTETp). In tube B (Fig. 1), we used the same $G$. pallida-specific set for the detection of $G$. pallida; however, we multiplexed it with the G. tabacumspecific set (primer PGrtf, primer PITSt3, and the TET-labeled TaqMan probe GTETp). The components for each multiplex assay in $25 \mu \mathrm{l}$ were $200 \mathrm{nM}$ each primer, $100 \mathrm{nM}$ each probe, $6.0 \mathrm{mM}$ $\mathrm{MgCl}_{2}, 240 \mu \mathrm{M}$ (each) dNTPs, 1× PCR buffer, and 1 unit of Platinum Taq DNA polymerase (Invitrogen, Carlsbad, CA). The cycling parameters included an initial denaturation step at $95^{\circ} \mathrm{C}$ for $120 \mathrm{~s}$ followed by 40 cycles at $95^{\circ} \mathrm{C}$ for $1 \mathrm{~s}$ and $58^{\circ} \mathrm{C}$ for $40 \mathrm{~s}$. Each run contained one positive control (for each species) and one negative control.

Conventional PCR protocol. Amplifications of ITS1 and ITS2 were performed on each nematode sample to confirm the DNA template quality for all populations included in this study. Negative controls containing water instead of DNA template were included with each experiment. PCR reactions contained $0.2 \mu \mathrm{M}$ each for primers TW81 (9) and AB28 (8), $5 \mu$ l of nematode DNA extract, $200 \mu \mathrm{M}$ (each) dNTPs, $1.5 \mathrm{mM} \mathrm{MgCl} 2,1.5$ units of Platinum Taq DNA Polymerase (Invitrogen), and supplied enzyme reaction buffer at $1 \times$ in a total volume of $50 \mu \mathrm{l}$. The Biometra TGradient cycling parameters included an initial denaturation step of $95^{\circ} \mathrm{C}$ for $120 \mathrm{~s}$; followed by 35 cycles of $95^{\circ} \mathrm{C}$ for $30 \mathrm{~s}, 55^{\circ} \mathrm{C}$ for $30 \mathrm{~s}$, and $72^{\circ} \mathrm{C}$ for $90 \mathrm{~s}$; and finished

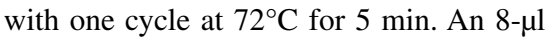
aliquot of each PCR reaction was analyzed by electrophoresis on $1 \%$ agarose in $1 \times$ Tris-acetate-EDTA. Gels were stained with ethidium bromide and visualized by UV illumination. Gel images were captured with a ChemiImager (Alpha Innotech, San Leandro, CA).

Establishment of standard curves. Conventional PCR products from three samples each of G. pallida, G. rostochien- 
sis, and G. tabacum were excised from agarose gels, purified with the QIAquick Gel Extraction Kit (Qiagen, Valencia, CA), and cloned into the pCR4-TOPO plasmid vector (Invitrogen). Plasmid DNA was purified using the QIAprep Spin Miniprep Kit (Qiagen) and the cloned DNA fragments were sequenced in both directions using the M13 forward (-20) and M13 reverse primers. Sequencing was performed at the University of Maryland Biotechnology Institute, Center for Biosystems Research (College Park, MD). Sequences were compared with sequences in GenBank of other Globodera populations. The BLASTN program (http://blast. ncbi.nlm.nih.gov/Blast.cgi) was used to determine the level of nucleotide similarity with other Globodera spp. The plasmids pGp, pGr, and pGt were selected for $G$. pallida, G. rostochiensis, and G. tabacum, respectively. To evaluate the amplification efficiency, assay accuracy, and low detection limits of the qPCR methods, an absolute standard curve was established for each of the three nematode species using the respective plasmid. The standard curves were based on 10-fold serial dilutions prepared in water from $1.00 \times 10^{7}$ copies per microliter of initial plasmid concentration.

\section{RESULTS}

DNA extraction efficiency. In the traditional single-worm smash extraction method, specially trained personnel physically separate juveniles with the aid of a dissecting microscope and slice individuals to liberate the DNA for extraction. In contrast, the rapid nematode-DNA extraction method was carried out in a closed-tube system using disposable microcentrifuge tubes and disposable glass beads. DNA extraction from 12 samples required about $2 \mathrm{~h}$ with no need for personnel trained in the manipulation of single nematodes or use of a microscope. The rapid nematodeDNA extraction method yielded highquality DNA suitable for PCR amplification from G. pallida, G. rostochiensis, and G. tabacum (Fig. 2). The PCR-amplified DNA bands were comparable with those produced from DNA extracted using the single-worm smash method. The yield and quality of DNA extracts were readily monitored by the cross threshold $(\mathrm{Ct})$ values of the multiplex qPCR. The $\mathrm{Ct}$ value is inversely related directly to the amount of the PCR product and, therefore, to the original amount of the nematode target DNA present in the PCR reaction. The lower the $\mathrm{Ct}$ value, the higher the nematode target DNA concentration in the nematode DNA extract. Of the $20 \mu \mathrm{l}$ of total DNA extracted from a single juvenile (using either the single-worm smash or the rapid nematode-DNA extraction methods), $2 \mu$ yielded stable qPCR Ct values, averaging 27.81, 31.99, and 28.28 for the tested populations of G. pallida, G. rosto- chiensis, and G. tabacum, respectively (Tables 1 and 4).

Primer and probe specificity. The consensus sequences of G. pallida, G. rostochiensis, and G. tabacum were aligned and analyzed. Based on the conserved nucleotide regions, a primer pair and a TaqMan probe were designed for the detection of each species (Table 3; Fig. 3). The specificity of each assay was evaluated in multiplex qPCR assays using DNA extracts of G. pallida, G. rostochiensis, G. tabacum, and other nematode species (Tables 1 and
4). As expected, each primer/probe set proved specific to each target, and nontemplate negative controls gave $0.00 \mathrm{Ct}$ values. None of the three primer/probe sets cross-reacted with other selected nematode species evaluated in this study (Table 1), including $H$. avenae, $H$. glycines, $H$. lespedezae, M. chitwoodi, M. hapla, M. naasi, N. aberrans, $P$. penetrans, and an unusual Globodera population of unknown host status from Oregon. The G. pallida primer/probe set, specific for $G$. pallida, reacted positively with all populations of

Table 2. List of sequences for GenBank accession numbers of the nematode species and populations used in the development of primers and probes during this study

\begin{tabular}{|c|c|c|}
\hline Species & Location & Accession no. \\
\hline \multirow[t]{17}{*}{ Globodera pallida } & United States & EF153835 \\
\hline & $\ldots$ & EF153836 \\
\hline & $\ldots$ & EF153837 \\
\hline & Canada & FJ212165 \\
\hline & $\ldots$ & GQ294522 \\
\hline & $\ldots$ & GQ294523 \\
\hline & $\ldots$ & GQ355975 \\
\hline & Peru & AF016866 \\
\hline & $\ldots$ & AF016868 \\
\hline & Chile & EF153834 \\
\hline & Argentina & DQ097514 \\
\hline & United Kingdom & DQ847109 \\
\hline & & AF016869 \\
\hline & Spain & AF016871 \\
\hline & Romania & AF016870 \\
\hline & Ukraine & AJ606687 \\
\hline & New Zealand & EF622533 \\
\hline \multirow[t]{11}{*}{ G. rostochiensis } & United States & EF153839 \\
\hline & United Kingdom & EF153840 \\
\hline & Russia & DQ847117 \\
\hline & $\ldots$ & DQ847119 \\
\hline & $\ldots$ & DQ847120 \\
\hline & Japan & AB207271 \\
\hline & Australia & EF622522 \\
\hline & $\ldots$ & EF622523 \\
\hline & $\ldots$ & EF622525 \\
\hline & $\ldots$ & EF622528 \\
\hline & $\ldots$ & EF622532 \\
\hline \multirow[t]{3}{*}{ G. tabacum solanacearum } & United States & DQ847114 \\
\hline & $\ldots$ & DQ847115 \\
\hline & & EF153841 \\
\hline \multirow[t]{3}{*}{ G. tabacum tabacum } & United States & EF153842 \\
\hline & & DQ847116 \\
\hline & Japan & AB207272 \\
\hline \multirow[t]{2}{*}{ G. tabacum virginiae } & United States & DQ847112 \\
\hline & $\ldots$ & DQ847113 \\
\hline G. achilleae & Slovenia & AY599498 \\
\hline G. artemisiae & China & AF274415 \\
\hline G. hypolysi & Japan & AB207273 \\
\hline G. millefolii & Estonia & AF161004 \\
\hline
\end{tabular}

Table 3. Sequence of TaqMan primers and probes for amplification of rDNA internal transcribed spacer I region of Globodera spp.

\begin{tabular}{|c|c|}
\hline Primer or probe name & $5^{\prime}$ to $3^{\prime}$ Sequence \\
\hline PITSpf $^{\mathrm{a}}$ & ACGGACACATGCCCGCTA \\
\hline PITSp4 ${ }^{\text {b }}$ & ACAACAGCAATCGTCGAG \\
\hline GFAMp $p^{a}$ & FAM/ACATGAGTGTTGGGGTGTAAC/BHQ-1 \\
\hline PGrtf ${ }^{a}$ & TCTGTGCGTCGTTGAGC \\
\hline Prostor $^{\mathrm{c}}$ & CGCAGACATGCCGCAA \\
\hline GTETp $^{a}$ & TET/CGCAGATATGCTAACATGGAGTGTAG/BHQ-2 \\
\hline Ptab-rt2fa & TCGTTGAGCGGTTGTTGC \\
\hline PITSt3mr ${ }^{\mathrm{d}}$ & AGCGCAGATATGCCGCG \\
\hline
\end{tabular}

${ }^{a}$ Developed for this study.

b Bulman and Marshall primer (5).

${ }^{\mathrm{c}}$ Modification of the primer PITSr3 (5).

${ }^{\mathrm{d}}$ Modification of the primer PITSt3 (23). 
pale potato cyst nematode tested, including those from the United States, Peru, Chile, the United Kingdom, Switzerland, and Netherlands. This set did not cross react with other tested Globodera spp. ( $G$. rostochiensis, G. tabacum, or the Globodera sp. from Oregon). The G. rostochiensis set, specific for $G$. rostochiensis, reacted positively with golden potato nematode populations from Canada, the United States, and the United Kingdom and did not cross react with other tested Globodera spp. (G. pallida, G. tabacum, and the Oregon Globodera sp.). Finally, the G. $t a$ bacum set, specific for G. tabacum, reacted positively with $G$. tabacum tabacum populations from Virginia and Connecticut and with a $G$. tabacum solanacearum population from Virginia. Similar to the $G$. pallida and $G$. rostochiensis sets, this set did not cross-react with any of the other tested Globodera spp. (Table 1).

Assay sensitivity. To evaluate the analytical detection sensitivity, four standard

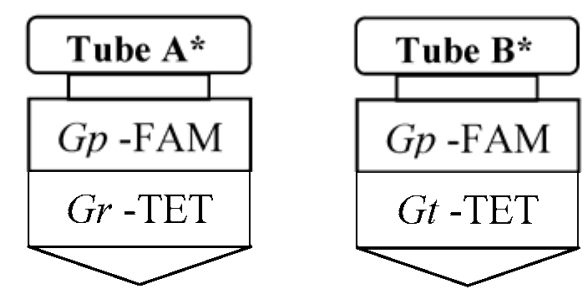

\begin{tabular}{|c|c|c|}
\hline Tube A & Tube B & \multicolumn{1}{c|}{ Results } \\
Reaction & Reaction & Interpretation \\
\hline FAM $+\&$ TET - & FAM $+\&$ TET - & G. pallida \\
\hline FAM - \& TET + & FAM - \& TET - & G. rostochiensis \\
\hline FAM - \& TET - & FAM - \& TET + & G. tabacum \\
\hline
\end{tabular}

Fig. 1. Multiplex quantitative polymerase chain reaction illustration displaying the use of two tubes for identification of three Globodera spp. ${ }^{*}$ Gp-FAM $=$ Globodera pallida-specific primer pair and TaqMan probe with FAM reporter dye; Gr-TET $=$ G. rostochiensis -specific primer pair and TaqMan probe with TET reporter dye; Gt-TET = G. tabacum-specific primer pair and TaqMan probe with TET reporter dye

Table 4. Average cross-threshold $(\mathrm{Ct})$ values for nematode samples extracted using rapid nematodeDNA extraction method

\begin{tabular}{|c|c|c|c|c|}
\hline \multirow[b]{3}{*}{ Species, sample description ${ }^{a}$} & \multicolumn{4}{|c|}{ Ct value } \\
\hline & \multicolumn{2}{|c|}{ Tube A } & \multicolumn{2}{|c|}{ Tube B } \\
\hline & FAM & TET & FAM & TET \\
\hline \multicolumn{5}{|l|}{ Globodera pallida } \\
\hline 1 cyst & 23.62 & 0 & 23.84 & 0 \\
\hline 100 juveniles & 23.05 & 0 & 22.93 & 0 \\
\hline 50 juveniles & 25.16 & 0 & 25.3 & 0 \\
\hline 10 juveniles & 26.43 & 0 & 26.28 & 0 \\
\hline 1 juvenile & 29.89 & 0 & 29.78 & 0 \\
\hline \multicolumn{5}{|l|}{ G. rostochiensis } \\
\hline 1 cyst & 0 & 24.59 & 0 & 0 \\
\hline 100 juveniles & 0 & 23.07 & 0 & 0 \\
\hline 50 juveniles & 0 & 25.93 & 0 & 0 \\
\hline 10 juveniles & 0 & 26.43 & 0 & 0 \\
\hline 1 juvenile & 0 & 30.33 & 0 & 0 \\
\hline \multicolumn{5}{|l|}{ G. tabacum solanacearum } \\
\hline 1 cyst & 0 & 0 & 0 & 24.33 \\
\hline 100 juveniles & 0 & 0 & 0 & 22.19 \\
\hline 50 juveniles & 0 & 0 & 0 & 24.70 \\
\hline 10 juveniles & 0 & 0 & 0 & 25.40 \\
\hline 1 juvenile & 0 & 0 & 0 & 28.75 \\
\hline
\end{tabular}

${ }^{a}$ Intact cysts were examined using a microscope to insure that they contained eggs or juveniles before DNA extraction. Other cysts were cracked open in molecular-biology-grade water and the exact numbers of juveniles required were transferred to microcentrifuge tubes for DNA extraction.

curves were established using the ITS1cloned plasmid DNA (Fig. 4). As expected from their primer and probe design, all three qPCR reactions for G. pallida, $G$. rostochiensis, and $G$. tabacum had very high assay accuracy $\left(R^{2}>0.99\right)$, with no significant differences found among them. All three PCR reactions also showed very high assay precision, as indicated by low standard deviations of the mean $\mathrm{Ct}$ values (standard deviation ranging from 0.03 to 0.33). The PCR amplification efficiency calculated from the slope of the standard curves (10) was 98.93, 86.51, and 88.54\% for $G$. pallida, $G$. rostochiensis, and $G$. tabacum, respectively. Based on their standard curves for absolute quantification, the analytical low detection limit was 1 to 5 copies of their target ITS1 templates per reaction. Based on average $\mathrm{Ct}$ values (Table 4) and the use of $2 \mu$ l of DNA extract (of $20 \mu \mathrm{l}$ total), the average ITS1 copy number per single juvenile calculated from standard curves was $6.97 \times 10^{4}, 8.34 \times 10^{2}$, and $7.44 \times 10^{4}$ copies for G. pallida, G. rostochiensis, and G. tabacum, respectively. There were no significant differences in the FAM Ct values for G. pallida between the two tests in tube $\mathrm{A}$ and tube $\mathrm{B}$, indicating that no significant influences on assay sensitivity or performance of the $G$. pallida set by the $G$. rostochiensis set in tube A or by the G. tabacum set in tube B occurred in the multiplex qPCR reactions.

Two-tube assay utility. The two-tube assay developed in this study allowed the detection and identification of G. pallida, $G$. rostochiensis, and G. tabacum simultaneously. Both PCN species were detected and identified using the multiplex reaction in tube $\mathrm{A}$ ( $G$. pallida was detected using FAM, whereas G. rostochiensis was detected using TET reporting dye; Table 4). Using the multiplex reaction in tube $\mathrm{B}, G$. pallida was detected using the FAM reporting dye, whereas G. tabacum was detected using TET.

Each sample of G. pallida twice tested positive with reporting dye FAM in both tube $\mathrm{A}$ and tube $\mathrm{B}$ of the two-tube system (Tables 1 and 4). Using single juveniles of

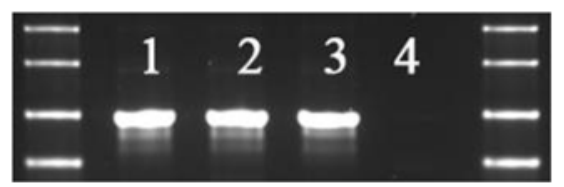

Fig. 2. Agarose gel electrophoresis displaying conventional polymerase chain reaction (PCR) reaction of Globodera spp. using the general primers TW81 and AB28. DNA was extracted using the rapid nematode-DNA extraction method. Outer lanes, BioMarker Ext DNA ladder (BioVentures, Inc., Murfreesboro, TN); lanes 1-3, PCR-amplified approximately $980-b p$ DNA fragments representing the ribosomal internal transcribed spacer (ITS) 1 and 2 of Globodera spp.; lane 1, Globodera pallida; lane 2, G. rostochiensis; lane 3, G. tabacum; lane 4, nontemplate negative control. 
G. pallida populations from Idaho, Switzerland, Peru, England, Netherlands, or Chile, the average FAM Ct value was 27.81. The average FAM Ct values for the Idaho population were $29.84,26.36,25.23,22.99$, and 23.73 for single, 10,50 , and 100 juveniles and 1 cyst, respectively (Table 4). All $G$. rostochiensis samples tested positive with the reporting dye TET in tube A. The average TET Ct value was 31.99 for the single juveniles tested from four populations (Table 1). For the G. rostochiensis population from New York, the average TET Ct values were $30.33,26.43,25.93,23.07$, and 24.59 for single, 10, 50, and 100 juveniles and 1 cyst, respectively (Table 4). All G. tabacum populations tested positive with the reporting dye TET in tube B. The average TET Ct value was 28.28 for single juveniles tested from six populations (Table 1). The Richmond, VA population TET Ct average values were $28.75,25.4,24.7,22.19$, and 24.33 for single, 10, 50, and 100 juveniles and 1 cyst, respectively.

\section{DISCUSSION}

The main focus of the PCN eradication program in the United States has been on detection of the pale potato cyst nematode (G. pallida) and limiting spread of the golden potato cyst nematode ( $G$. rostochiensis). As part of this effort, federal and state agencies have conducted field surveys of potato-growing acreage to determine the extent of possible PCN infestations. In some cases, the poor condition of the specimens obtained from field samples has precluded easy identification of the nematodes based on cyst or juvenile morphology. Based on morphological characterization, it can be extremely challenging to differentiate G. pallida, a potato cyst

\section{A G. pallida detection}

\begin{tabular}{|c|c|c|c|c|c|c|}
\hline & & Primer PITSPf & & Probe GFAMP & & Primer PITSPA \\
\hline Gpal & cons & ACGGACACATGCCCGCTA & $\mathrm{xxx}$ & ACATGAGTGTTGGGGTGTAAC & $\mathrm{x} x \mathrm{x}$ & CTCGACGATTGCTGTTGT \\
\hline$\sigma_{F}$ & Arg & ACGGACACATGCCCGCTG & $\mathrm{xxx}$ & ACATGAGTGTT & $x \times x$ & SCTGT \\
\hline Gro: & cons & ACGGACAYATGCCCRCTG & $\mathrm{xxx}$ & ACATGAGTGTTGGGGTRTAAC & $\mathrm{xxx}$ & GCCGACGATTGCTGCTGT \\
\hline o. & cons & ACGGACACATGCCCGCTG & $\mathrm{xxx}$ & ACATGAGTGTTGGGGTGTAAC & $\mathrm{xxx}$ & GCCGACGATTGCTGCTNT \\
\hline Gach & & ACGGACACATGCCCGTTG & $\mathrm{xxx}$ & ACAAGAGTTTTGGGGTGTTAC & $\mathrm{xxx}$ & GCCGACGATTGCTGTCAT \\
\hline Ghyp & & ACGGACACATGCCCGTTG & $\mathrm{XXX}$ & ACAAGAGTTTTGGGGTGTTAC & $\mathrm{xxx}$ & GCCGACGATTGCTGTCGT \\
\hline & & ACACATGCCCGTTG & $\mathrm{xxx}$ & AGAGTTTTGGGGTGTTAC & $\mathrm{xxx}$ & GATTGCTGTCGT \\
\hline 1 & & ACGGGCACAGGCCCTACG & $\mathrm{xxx}$ & GCACTAGT. TTGGGGCGCTAC & $\mathrm{xxx}$ & . GCTGCTGC \\
\hline$o b$ & cons & ACGGRCAYAKGCCCDHYR & $\mathrm{xxx}$ & RCAHKAGTKTTGGGGYRYWAC & $\mathrm{xxx}$ & SYCGACRATTGCTGYYNY \\
\hline
\end{tabular}

B G. rostochiensis detection

\begin{tabular}{|c|c|c|c|c|c|c|}
\hline & & Primer PGrtf & & Probe GTETP & & $\begin{array}{l}\text { Primer Prostor } \\
\text { CRGCGGCATRTCTGCG }\end{array}$ \\
\hline Gpal & $\mathrm{n}$ & TCTGTGCGTCGTTGAGC & $\mathrm{xx}$ & CGSBG. ATATGCTGACATGGAGTG. . TAG & $x x$ & $\mathrm{CRG}$ \\
\hline al & A] & TCTGTGCGTCGTTGAGC & $\mathrm{xx}$ & TG. . TAT & $\mathrm{xx}$ & \\
\hline Gros & cons & TCTGTGCGTCGTTGAGC & $\mathrm{xx}$ & CGCAG. ATATGCTAACATGGAGTG. . TAG & $\mathrm{xx}$ & 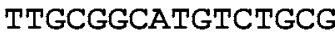 \\
\hline & cons & TCTGTGCGTCGTTGAGC & $\mathrm{xx}$ & CGCGG.ATATGCTAACATGGAGTG . TAN & $\mathrm{xx}$ & $\mathrm{CCGC}$ \\
\hline Gach & & TCTGTGCGTCGTTGAGC & $\mathrm{xx}$ & CGCTG.ATATGCTGACATGGAGTGTGTAG & $\mathrm{xx}$ & TGGCGGCATGTC \\
\hline Ghyp & & TCTGTGCGTCGTTTGAG & $x \mathrm{x}$ & CGCTG.ATATGCTGACATGGAGTGTGTAG & $\mathrm{xx}$ & GGCATGTC \\
\hline t & & TCTGTGCGT & $\mathrm{xx}$ & GCTG.ATATGCTGACATGGAGTGTGTAG & $\mathrm{xx}$ & TG \\
\hline & & TCTGTGCGTCGTTGAGT & $\mathrm{xx}$ & CGTGGGACATACTGATGTGTAAGTGTTGT & $\mathrm{xx}$ & GGTATGTC \\
\hline$a b$ & & TCTGTGCGTCGTTGAGB & $x x$ & BNG . AYATRCTRAYRTGKARKK . . TRN & $x x$ & YDC \\
\hline
\end{tabular}

\section{G. tabacum detection}

\begin{tabular}{|c|c|c|c|}
\hline & Primer PGrtf & Probe GTETP & Primer PITSt3mr \\
\hline bal & TCTGTGCGTCGTTGAGC & XX CGSBG.ATATGCTGACATGGAGTG. .TAG & XX RGCGGCATRTCTGCGCY \\
\hline 1 Arg & TCTGTGCGTCGTTGAGC & $\mathrm{XX}$ CGCG & $\mathrm{xx} A$ \\
\hline is con. & CGTTGAGC & GGAGIG. .IAG & $\mathrm{XX} \mathrm{TG}$ \\
\hline & TCTGTGCGTCGTTGAGC & XX CGCGG. ATATGCTAACATGGAGTG. . TAK & $\mathrm{x} \times \mathrm{CGC}$ \\
\hline & TCTGTGCGTCGTTGAGC & GCTG.ATATGCTGACATGGAGTGTGTAG & XX GGCGGCATGTCGGCGTT \\
\hline & TCTGTGCGTCGTTTGAG & xX CGCTG.ATATGCTGACATGGAGTGTGTAG & xx GGCGGCATGTCGGCGTT \\
\hline & TCTGTGCGTCGTTGAGC & GCTG.ATATGCTGACATGGAGTGTGTAG & $\mathrm{xX}$ GGCGGCATGTCGGCGTT \\
\hline & CTGTGCGTCGTTGAGT & GTGGGACATACTGATGTGTAAGTGTTGT & XX AGCGGTATGTCTGTGCT \\
\hline & CTGTGCGTCGTTGAGB & $x X$ CGBNG.AYATRCTRAYRTGKARKK. . TRN & XX NGCGGYATRTCKGYGYY \\
\hline
\end{tabular}

Fig. 3. TaqMan quantitative polymerase chain reaction target sequences (lines in bold) aligned with sequences from other Globodera spp. (sequence accession numbers listed in Table 2). A, Globodera pallida detection. B, G. rostochiensis detection. C, G. tabacum detection. Gpal cons = G. pallida sequence consensus (16 sequences, representing populations from the United States, Canada, the United Kingdom, New Zealand, Spain, Romania, Peru, Chile, and Ukraine); Gpal Arg = G. pallida sequence from Argentina; Gros cons = G. rostochiensis sequence consensus [11 sequences, representing populations from the United States, the United Kingdom., Russia, Japan, and Australia); Gtab cons = G. tabacum sequence consensus (G. tabacum solanacearum from the United States); G. tabacum tabacum from the United States and Japan; G. tabacum virginiae from the United States; Gach = G. achilleae sequence from Slovenia; Ghyp $=$ G. hypolysi sequence from Japan; Gart $=$ G. artemisiae sequence from China; Gmil $=$ G. millefolii sequence from Estonia; Glob cons $=$ consensus sequence. Bases that were ambiguous or mismatched the probe sequences are indicated in bold for nontarget species. $\mathrm{Y}=\mathrm{C}, \mathrm{T} ; \mathrm{R}=\mathrm{A}, \mathrm{G} ; \mathrm{W}=\mathrm{A}, \mathrm{T}$; $\mathrm{S}=\mathrm{C}, \mathrm{G} ; \mathrm{K}=\mathrm{G}, \mathrm{T} ; \mathrm{D}=\mathrm{G}, \mathrm{T}, \mathrm{A} ; \mathrm{B}=\mathrm{C}, \mathrm{G}, \mathrm{T} ; \mathrm{H}=\mathrm{A}, \mathrm{C}, \mathrm{T} ;$ and $\mathrm{N}=\mathrm{A}, \mathrm{G}, \mathrm{C}, \mathrm{T}$. 
nematode, from G. tabacum, the closely related tobacco cyst nematode. The existence of three G. tabacum subspecies of overlapping morphology further complicates morphological identification. Although molecular diagnostic assays capa- ble of distinguishing $G$. pallida, $G$. rostochiensis, and $G$. tabacum have been developed recently (23), these conventional PCR assays take 1 to 2 days to complete.

A number of $\mathrm{qPCR}$ assays have been developed for Globodera spp. detection
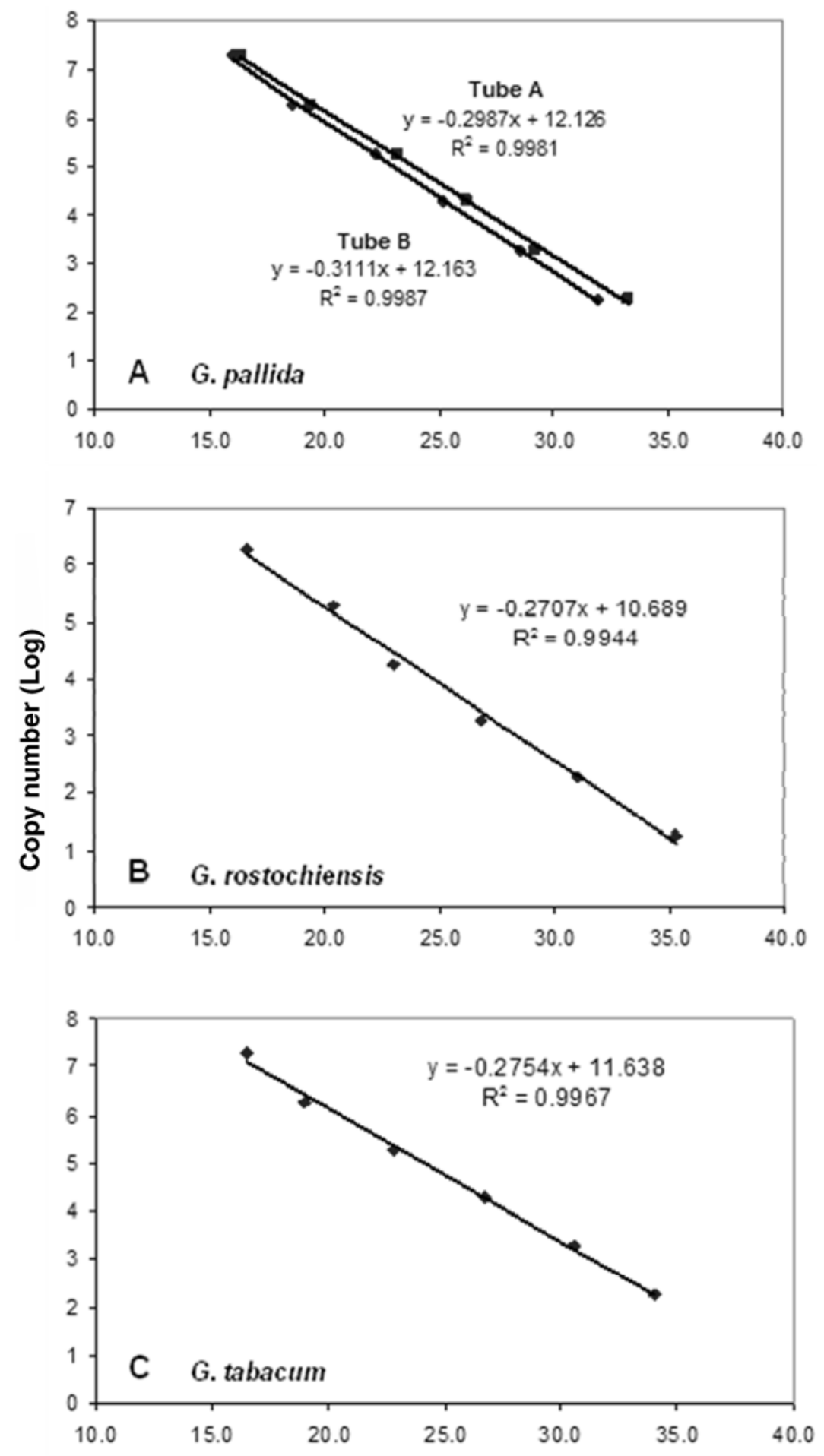

\section{Mean CT values of real-time PCR}

Fig. 4. Standard curves of the quantitative polymerase chain reaction (qPCR) for detection of A, Globodera pallida; B, G. rostochiensis; and C, G. tabacum. Standard curves were established on the mean cross-threshold $(\mathrm{Ct})$ values of 10 -fold serial dilutions of template-cloned plasmid DNA in triplicates in tube A for G. pallida and G. rostochiensis and tube B for G. pallida and G. tabacum, in the two-tube system of multiplex qPCR.

but none of these studies have included all species of regulatory concern in Canada, United States, Mexico, and Central America. Bates et al. (3) employed melting-peak analysis of ITS PCR products, generated with the Bulman-Marshall primers (5) and detection with SYBR Green I fluorescent dye to identify the relative proportion of $G$. pallida and G. rostochiensis within mixedcyst samples. Subsequent work by Quader et al. (22) focused on improving DNA extraction methods to give more consistent PCR using a commercial kit rather than phenol-chloroform extraction, and the associated qPCR assay also employed SYBR Green I dye for detection of ITS PCR products. Their study demonstrated that a broad geographic range of $G$. rostochiensis populations was successfully detected by those primers, focusing on species of primary concern in Australia and New Zealand. A TaqMan assay using ITSbased primers and probes was also developed to distinguish $G$. rostochiensis, a pest of regulatory concern in Europe, from $G$. artemisiae, which is not a parasite of potato (19).

In this study, the combination of rapid nematode-DNA extraction with multiplex qPCR resulted in a robust detection and identification protocol available for widescale surveys and for the diagnostic confirmation of PCN-suspect samples. Molecular identification of PCN suspect samples was completed within about $4 \mathrm{~h}$ from the time of delivery to the diagnostic laboratory because we eliminated the need to use a microscope or perform and analyze conventional PCR. Furthermore, the use of qPCR assays minimized laboratory exposure to concentrated PCR products, a potential source of contamination that can confound accurate diagnostics.

As predicted from the primer design alignments, G. pallida was successfully identified using the specific PITSpf forward primer paired with the speciesspecific PITSp4 (5) reverse primer. One $G$. pallida sequence from Argentina contained one mismatch in PITSpf and three mismatches in PITSp4. Reaction of the $G$. pallida-specific primers with this population could not be tested directly, because the Argentina population was not available. However, subsequent to the timing of this study, an unnamed Globodera population from Oregon was found to have ITS sequences that are highly similar to the Argentina population and also contain mismatches to the $G$. pallida primer set (not shown). As expected, qPCR results from this primer set with the Oregon population were negative, as they were with the other Globodera spp. tested. Although the population was not available to be tested directly, ITS sequences from the as-yetunvalidated species G. "mexicana" (GenBank accession numbers EU006707, 
EU006708, and EU006709) were compared with the primer and probe sequences. Except for one base mismatch with pITSp4, the G. "mexicana" sequences matched the G. pallida-specific primer/ probe combination.

In combination with the common forward primer PGrtf, the reverse primer Prostor (a modification of the Bulman \& Marshall PITSr3 primer; 5) was used for the specific detection of $G$. rostochiensis, while the specific reverse primer PITSt $3 \mathrm{mr}$ (a modification of primer PITS3; 23) was used for the specific detection of $G$. tabacum. The GTETp probe was designed to anneal to G. rostochiensis, G. tabacum, and $G$. pallida but not to $G$. achilleae, $G$. hypolysi, G. artemisiae, and G. millefolii because the target sequences of these latter Globodera spp. each have two nucleotides extra in the target sequence of the probe. As predicted, by combining the gap in the probe and the $3^{\prime}$ end specificity of the reverse primers, both $G$. rostochiensis and G. tabacum were successfully detected and identified using the G. rostochiensis- and G. tabacum-specific sets, respectively. These primer sets also reacted negatively with the Oregon population.

Primer/probe sets that were specific to G. pallida, G. rostochiensis, or G. tabacum showed promising assay sensitivity, yielding positive mean $\mathrm{Ct}$ values between 27 to 32 when using $2 \mu$ l of the single juvenile DNA extracts from the three nematode species. The analytic low detection limits for the three primer/probe sets were as low as one to five copies of the DNA template per reaction. In addition, the qPCR sensitivity remained the same in the multiplex assay compared with the singleplex qPCR assay. As expected, the qPCR Ct values dropped linearly and accordingly with the increase of the number of juvenile nematodes used per extraction. The multiplex qPCR yielded $\mathrm{Ct}$ values around 24 for single cysts of the three Globodera spp. evaluated.

The two-tube multiplex qPCR system developed in this work could detect, identify, and quantify even a single juvenile of the three Globodera spp. most likely to be encountered during North American surveys for PCN. It is realistic to expect that the future may bring new diagnostic challenges as additional PCN populations are described both morphologically and molecularly. G. pallida originating from the cordillera of South America is of particular interest, considering the genetic diversity of those populations relative to the majority of
European and North American populations $(20,21,23,24)$. It is expected that development of PCN diagnostic protocols will continue to evolve as information about new populations is revealed.

\section{ACKNOWLEDGMENTS}

We thank M. Hult and S. Ochs for excellent technical assistance, and R. Bulluck and D. Chitwood for critical reading of the manuscript.

\section{LITERATURE CITED}

1. Bačić, J., Stare, B. G., Sirrca, S., and Urek, G. 2008. Analyses of Globodera rostochiensis and G. pallida populations from Serbia by morphometrics and real-time PCR. Russ. J. Nematol. 16:63-65.

2. Baldwin, J. G., and Mundo-Ocampo, M. 1991. Heteroderinae, Cyst- and non-cyst-forming nematodes. Pages 275-362 in: Manual of Agricultural Nematology. W. R. Nickle, ed. Marcel Dekker, New York.

3. Bates, J. A., Taylor, E. J. A., Gans, P. T., and Thomas, J. E. 2002. Determination of relative proportions of Globodera species in mixed populations of potato cyst nematodes using PCR product melting peak analysis. Mol. Plant Pathol. 3:153-161.

4. Bélair, G., and Miller, S. 2006. First report of Globodera tabacum infecting tobacco plants in Quebec, Canada. Plant Dis. 90:527.

5. Bulman, S. R., and Marshall, J. W. 1997. Differentiation of Australasian potato cyst nematode (PCN) populations using the polymerase chain reaction (PCR). N. Z. J. Crop Hortic. 25:123-129.

6. den Nijs, L., and Karssen, G. 2004. Diagnostic protocols for regulated pests, Globodera rostochiensis and Globodera pallida. EPPO Bull. 34:309-314.

7. Hafez, S. L., Sundararaj, P., Handoo, Z. A., Skantar, A. M., Carta, L. K., and Chitwood, D. J. 2007. First report of the pale cyst nematode, Globodera pallida, in the United States. Plant Dis. 91:325.

8. Howlett, B. J., Brownlee, A. G., Guest, D. I., Adcock, G. J., and McFadden, G. I. 1992. The 5S ribosomal RNA gene is linked to large and small subunit ribosomal RNA genes in the oomycetes, Phytophthora vignae, P. cinnamomi, P. megaspera f. sp. glycinae and Saprolegnia ferax. Curr. Genet. 22:455-461.

9. Joyce, S. A., Reid, A., Driver, F., and Curran, J. 1994. Application of polymerase chain reaction (PCR) methods to identification of entomopathogenic nematodes. Pages 178-187 in: COST 812 Biotechnology: Genetics of Entomopathogenic Nematode-Bacterium Complexes. A. M. Burnell, R.-U. Ehlers, and J. P. Masson, eds. Proc. Symp. Workshop, St. Patrick's College, Maynooth, County Kildare, Ireland. European Commission, DG XII, Luxembourg.

10. Li, W., Li, D., Twieg, E., Hartung, J. S., and Levy, L. 2008. Optimized quantification of unculturable Candidatus Liberibacter spp. in host plants using real-time PCR. Plant Dis. 92:854861.

11. Machay I. M., Ardeb, K. E., and Nitsche, A. 2002. Survey and summary/real-time PCR in virology. Nucleic Acids Res. 30:1292-1305.

12. Madani, M., Subbotin, S. A., and Moens, M. 2005. Quantitative detection of the potato cyst nematode, Globodera pallida, and the beet cyst nematode, Heterodera schachtii, using real-time PCR with SYBR green I dye. Mol. Cell Probes 19:81-86.

13. Marché, L., Valette, S., Grenier, E., and Mugniéry, D. 2001. Intra-species DNA polymorphism in the tobacco cyst-nematode complex (Globodera tabacum) using AFLP. Genome 44:941-946.

14. Miller, L. I., and Gray, B. J. 1972. Heterodera solanacearum $\mathrm{N}$. sp., a parasite of solanaceous plants. Nematologica 18:404-413.

15. Mota, M., and Eisenback, J. D. 1993. Morphology of second-stage juveniles and males of Globodera tabacum tabacum, G. t. virginiae, and G. t. solanacearum (Nemata: Heteroderinae). J. Nematol. 25:27-33.

16. Mota, M., and Eisenback, J. D. 1993. Morphology of females and cysts of Globodera tabacum tabacum, G. t. virginiae, and G. t. solanacearum (Nemata: Heteroderinae). J. Nematol. 25:136-147.

17. Mota, M., and Eisenback, J. D. 1993. Morphometrics of Globodera tabacum tabacum, $G$. t. virginiae, and G. t. solanacearum (Nemata: Heteroderinae). J. Nematol. 25:148160.

18. Nakhla, M. K., Owens, K. J., Carta, L., Skantar, A., and Levy, L. 2007. Validation of potato cyst nematode (PCN) molecular identification methods and development of laboratory work instructions for a national survey. (Abstr.) Phytopathology 97:S82.

19. Nowaczyk, K., Dobosz, R. Kornobis, S., and Obrepalska-Steplowska, A. 2008. TaqMan REAL-Time PCR-based approach for differentiation between Globodera rostochiensis (golden nematode) and Globodera artemisiae species. Parasitol. Res. 103:577-581.

20. Plantard, O. Picard, D., Valette, S., Scurrah, M, Grenier, E., and Mugniéry, D. 2008. Origin and genetic diversity of Western European populations of the potato cyst nematode (Globodera pallida) inferred from mitochondrial sequences and microsatellite loci. Mol. Ecol. 17:2208-2218.

21. Pylypenko, L. A., Phillips, M. S., and Blok, V. C. 2008. Characterisation of two Ukrainian populations of Globodera pallida in terms of their virulence and mtDNA, and the biological assessment of a new resistant cultivar Vales Everest. Nematology 10:585-590.

22. Quader, M., Nambiar, L., and Cunnington, J. 2008. Conventional and real-time PCR-based species identification and diversity of potato cyst nematodes (Globodera spp.) from Victoria, Australia. Nematology 10:471-478.

23. Skantar, A. M., Handoo, Z. A., Carta, L. K., and Chitwood, D. J. 2007. Morphological and molecular identification of Globodera pallida associated with potato in Idaho. J. Nematol. 39:133-144.

24. Subbotin, S. A., Halford, P. D., Warry, A., and Perry, R. N. 2000. Variations in ribosomal DNA sequences and phylogeny of Globodera parasitizing solanaceous plants. Nematology 2:591-604.

25. Syracuse, A. J., Johnson, C. S., Eisenback, J. D., Nessler, C. L., and Smith, E. P. 2004. Intraspecific variability within Globodera tabacum solanacearum using random amplified polymorphic DNA. J. Nematol. 36:433-439.

26. Thomas, W. K., Vida, J. T., Frisse, L. M., Mundo, M., and Baldwin, J. G. 1997. DNA sequences from formalin fixed nematodes: Integrating molecular and morphological approaches to taxonomy. J. Nematol. 29:250254. 